\title{
Analysis on Crisis Management of Tourism Destination
}

\author{
Nan Chen, Aihua Su \\ Nanchang Institute of Science \& Technology, Nanchang, Jiangxi, 330000
}

\begin{abstract}
Tourism crisis research is an emerging research field in China, which involves cross-disciplinary research, including crisis theory, tourist behavior theory, human-earth relationship theory and so on. Especially in the tourism crisis occurs frequently today, the tourism crisis has become an important factor affecting the sustainable development of tourism destination, so the tourist destination must strengthen the study of tourism crisis.
\end{abstract}

Keywords: Crisis Management, Tourism Destination, Analysis

\section{Introduction}

Many tourism crisis has great influence on the tourism industry. It is necessary to take into account the impact of the tourism crisis on the tourists' destination choice during the planning and management of tourism destination. And be prepared in advance, once the advent of the tourism crisis can quickly start the crisis response program. This paper aims to make some contribution to this, that is to stress the tourism crisis on the tourism destination sustainable development of the major damage, hoping to cause the attention of tourist destination management, take seriously at any time may come to the tourism crisis can be well- The crisis response of tourists and the resulting choice of destination behavior to reveal the transmission mechanism after the tourism crisis, and to construct the crisis management of tourism destination. Finally, Mechanism is to help the tourism destination management agencies to effectively manage the crisis and reduce the losses caused by the crisis. 


\section{Analysis of Tourists' Perceptions of Tourism Crisis in Two Countries}

Crisis cognition is the subjective feeling and cognition of the individual to the various external crisis events, and these subjective feelings are influenced by many factors such as psychological, social and cultural factors. Slovic [62] proposed a psychometric model from the psychological point of view, summed up the impact of crisis awareness of the important dimensions and characteristics. Slovic argues that the judgment of a variety of crisis events can be perceived as "unknown, uncontrollable" from the "risk of anxiety", ie, perceived as "hard to control" and "unknown risk" Categories. In the factor space of these two factors, each crisis event has a relative position, and its position can directly show people's perceptual characteristics of the crisis.

There are many factors that cause the tourists to fear the tourism crisis and avoid the crisis. There are many crisis events, especially the crisis event with large-scale destruction effect, which brings about the terror and easy to personal and property safety. Severe threat is an important factor in tourism crisis avoidance. It is also an important factor that we usually think. However, the survey found that this is an important factor to avoid the crisis, but it is not the main factor. In other words, tourists avoid the crisis The main reason is not the fear of the event itself, listed in the influencing factors, $31.4 \%$ of the respondents chose the tourism crisis "destroyed the tourism experience", followed by $24.7 \%$ of the respondents chose " Can not control and deal with the crisis. " The pursuit of travel experience beyond reproach, this is the purpose of tourists and motivations. Leaving aside this factor, the overwhelming majority of tourists show the unpredictability of the outbreak of crisis and can not cope with and deal with the deep unease, it is this awareness of the crisis, to a large extent affected the tourists Travel decisions, in order to foolproof, choose a conservative strategy. According to the survey data show that adhere to the outbreak or the outbreak of the crisis is about the ratio of male to female tourists: $6: 1$, that is only a number of men with high adventurous choice to go. In the crisis, the development process, because the uncontrollable nature of the crisis, is caused by an important reason for psychological panic.

Tourists have some common psychological characteristics and needs, but specific to each person, there must be differences. Because of their different interests, abilities, temperament and personality, tourists constitute their own personality characteristics, so there are some differences in cognitive activities, emotional activities and intention activities. The tourists 'preferences affect the tourists' And the choice of tourism types, the impact of tourism preferences on tourists' perception of crisis can be studied from two perspectives.

Which try to experience the motive. It refers to the pursuit of new, strange, different, the United States, special feelings, to meet the special interests and hobbies generated by the tourism motive. Such as cave exploration, mountain climbing, rafting, skiing, cross-country, cycling tourism. Such tourists have a clear tendency to take risks, willing to accept the challenges of the crisis, the tourism crisis awareness is low. At the same time, tourists with such motivations 
often become volunteers in crisis relief and post-disaster reconstruction. Religious tourism motivators are also prone to crisis perception because of their religious beliefs, especially the crisis of religious holy places. Pious beliefs make them do not care about any difficulties and dangers, and are not afraid of the threat of any crisis.

\section{Travelers' skills to cope with the tourism crisis}

A large number of examples show that tourists with abundant experience in crises and sufficient skills can use their learned knowledge and relevant skills to protect themselves and help others. This type of tourists have a relatively low awareness of the crisis. Choice of destination is less affected by the crisis, even if the tourist destination to withstand the test of the crisis, it will not cause too much psychological trauma, they are proud to successfully deal with the crisis. The survey reflects a concern that the crisis management skills training that our tourists receive is very limited. Thirty-two respondents received training in emergency care or related crisis management $(14.5 \%$ of the total), and 188 $(85.5 \%)$ of the total did not receive any training Any form of relevant training.

Material resources: mainly for tourists in the crisis response can be used in materials, facilities and natural resources, after the crisis is often some of the crisis response directly or indirectly related to materials, facilities and resources have a special needs, and The market generally can not provide these substances in a timely manner, if tourists can not timely access to these material resources, their ability to respond will be greatly reduced. Social relief system: such as social security system, social and economic relief, evacuation and rescue plan, psychological assistance, legal aid, are crisis situations to cope with tourism crisis to extricate themselves from various external relief resources. If tourists have insufficient confidence in the external resources available to tourists in crisis situations, it will affect the perception of tourism crisis.

The easy-to-obtain strategy indicates that the individual can recognize and judge the current events mainly through the information easily extracted in the memory. Therefore, when the individual judges the frequency or probability of an event, it is easy to be detected by some newly-occurring, Interference. This is a bloody traffic accident will make most people think that the death rate of traffic accidents is higher than the cause of lung cancer mortality. Or why the "SARS" epidemic despite the mortality rate is far lower than traffic accidents and other accidents caused by the death rate, but it caused great fear throughout the world, is precisely because of our "SARS" high alert, Taken adequate precautions to prevent the spread of the epidemic, but can not be denied that the "SARS" epidemic has led to a lot beyond the capacity of social tolerance unnecessary fear.

Representational strategy refers to the fact that the individual always perceives the event based on some main characteristics. The study finds that people are more sensitive to the information of the symbolic information than the basic rate information, that is, the information about the similarity of things is easier to pay attention to. The underlying rate information that predicts the probability of 
occurrence of an event is more easily overlooked. This crisis of tourists cognitive strategy, so that the crisis in the gossip and rumors with the spread of the market, tourists tend to believe they have rather believe it has no attitude, believe the grapevine, exaggerating the impact of the crisis. Which objectively exacerbated the tourism crisis on the destructive impact of tourist destinations.

In the cognitive process is quite universal, it shows that an unrelated, preconceived figures or other things of any nature, are likely to become the basis for people's next cognitive and judgments, if there is a large deviation of the foundation, it is recognized Knowledge of the resulting bias is inevitable.

\section{Tourism Destination Crisis Management Mechanism}

Threatening prevention, do not see the outbreak of tourism crisis symptoms, from the tourist destination point of view all out of the normal state, but the normal is not eternal. Crisis is brewing in the calm outbreak. Crisis is inevitable, this time to strengthen the study of the crisis, prior to take the organization, management, diplomatic and other measures for the effective management of the tourism crisis is of great significance. Tourism destination in the crisis prevention stage is effective, in place will affect the next crisis management effect.

Pre-Crisis Preparedness The harbinger of the crisis has emerged, and experienced travel destination management should begin to prepare for crises. A number of examples show that this is an important turning point, and the preparations made during this period have a direct impact on the ability and effectiveness of tourism destinations to cope with crises during the crisis.

The timing of the crisis management program is very important, too early is not necessary, late will have a negative impact on tourism destination crisis management. The need for managers in the possession of sufficient information on the basis of decisive decision-making, timely start pre-designed crisis management program, because the form of crisis, the scale can not be predicted in advance, so the crisis management program must also maintain sufficient flexibility at any time according to the situation changes do Adjust accordingly.

The asymmetry of crisis information is the normality of information transmission in tourism crisis. Tourism crisis, so that tourists are not familiar with, uncontrollable crisis information is leading to psychological disturbances and excessive tension of the main reasons. Therefore, tourism destination management agencies in the crisis management is an important task is to break through the effective communication of information asymmetry. Timely and comprehensive dissemination of crisis information to the public (tourists), to tell the real situation of the crisis and to guide how to deal with the crisis, reduce the public (tourists) nervous and psychological pressure.

The first is to assess the material and economic loss of the destination, assess the devastating consequences of the crisis, and assess the loss in detail so as to provide the necessary information for the subsequent crisis recovery and reconstruction of the destination. Assessment of tourists in the past crisis in the 
cognitive and behavior, in order to support the crisis of tourists psychological intervention and the re-establishment of tourist destination image.

\section{Conclusion}

In this paper, we analyze the influencing factors of tourists' tourism crisis, the characteristics of information transmission in crisis situations and the limited rationality of tourists in crisis, and establish the crisis transmission mechanism of tourism destination. Analysis, I found that the impact of the crisis on the tourists is not dependent on its own destructive consequences, more important because the tourists do not understand the crisis, the tourists feel the crisis can not control, tourism, crisis response skills are limited As well as tourists and tourist destinations to communicate poor information and other reasons. It clarifies the bounded rationality of the tourists in the crisis, and points out that the tourism destination management institution must fully understand this point and make full and worst preparation in advance.

\section{References}

[1] Hong Jin. Calculation of information symmetry in crisis communication . Journal of Beijing Institute of Technology, 4(2), pp. 37-41, 2004.

[2] Fang Bangdao. Analysis of the motivation of tourism motivation. Journal of Kaifeng Institute of Education, 6(1), pp. 83-89, 2005.

[3] Zhou Xiaohong. Transformation Distortion: A Social Psychological Analysis of SARS Rumors . Sociological Research, 6(2), pp. 20-25, 2003.

[4] Shu Boyang, Yuan Jirong. Government-led and tourism destination image promotion research. Journal of Guilin Institute of Tourism. Journal of Guilin Institute of Tourism, 12(2), pp. 12-16, 2015.

[5] Shi Kan, Chen Xuefeng, Hu Weipeng. Study on risk cognition of SARS in Beijing . Population Research, 4(3), pp. 14-25, 2003. 